\title{
The Perception of Cultural Differences in Online Self-presentation
}

\author{
Yifan Jiang, Oscar de Bruijn, and Antonella De Angeli \\ Manchester Business School, Booth Street West, Manchester, M15 6PB, UK \\ Yifan.Jiang@postgrad.mbs.ac.uk, \\ \{Oscar.DeBruijn, Antonella.De-angeli\}@mbs.ac.uk
}

\begin{abstract}
Online self-presentation, defined as the way people present themselves through profiles, blogs, photo albums, etc., forms the basis of much of the interpersonal relationship building taking place in social networking platforms such as Windows Live Space. However, little is known about how people make sense of this information, particularly if presenter and audience do not have a common cultural background. This study investigated the effectiveness of cross-cultural online communication by measuring the cross-cultural social perception of specially constructed online representations of a typical British and a typical Chinese person. The representations were based on a 7-dimensional characterization of cultural differences derived from a review of the literature. The findings suggested that cultural characterization embedded in online communication affects the social perception of others, that it can trigger stereotypes, and that it has consequences for establishing relationships. Implications for the design of social networking platforms are discussed.
\end{abstract}

Keywords: Cross-cultural communication, Online Self-presentation.

\section{Introduction}

Social networking platforms (e.g., Windows Live Spaces, Facebook) provide an opportunity for users to present themselves online and connect with each other [5]. These media have the potential, therefore, to substantially increase the amount of communication between people who do not share a cultural background. However, it is questionable whether much of this cross-cultural communication is effective in establishing relationships between people with different cultural backgrounds. Indeed, cross-cultural relationships are "onerous to initiate, develop and maintain resulting from the interplay of a wide range of variables such as values, interest, personality traits, network patterns, communication styles, cultural knowledge, and relational and intercultural communication competence, intergroup attitudes, and so forth" [2].

Our cultural background serves to help us interpret our own behaviour and that of others. Thus, culture determines in large part how we present ourselves to the outside world, and how we perceive others. Self-presentation is subject to different levels of intentional control. Verbal communication can be more closely controlled, we can decide what to disclose and how, whereas non-verbal communication (e.g., physical appearance, gestures, tone of voice, and other behaviour) may escape conscious 
control. The way we are perceived by others will depend on the cues that they pick up from our verbal and non-verbal behaviour [7].

Online self-presentation is different from real life self-presentation. In online social networking sites, initial impressions of others are almost always based upon information they provide in their profiles, blogs and other forms of communication. These do not contain many of the non-verbal cues that are characteristic of offline communication. They are also substantially less spontaneous [11] [17], more static, and less immediately responsive to feedback. Evidence suggests that people adapt their style of self-presentation in offline communication according to the type of audience they are addressing [23]. The extent to which this can be achieved in online communication is often severely limited by the lack of affordances towards that end offered by online networking platforms. As a result, online self-presentation tends to emphasize a number of small cues based upon expectations of how audiences will react to these [4].

It appears therefore that the success of online communication for relationship building depends on the ability to anticipate the effects self-presentation has on others [15]. However, it seems likely that the perception of online self-presentation is substantially affected by the cultural background of the audience. In cross-cultural communication, therefore, it is unavoidable that presenters' anticipation of the effects of self-presentation is often inaccurate, as it is based on a cultural point of view that can be substantially different from that of the perceiver. In order to determine how cultural differences affects social networking, we have to investigate how cultural variation in self-presentation strategies affects the way people are perceived by others, and how these perceptions might affect cross-cultural encounters in online contexts.

\section{Background}

An important psychological framework, particularly relevant to the aim of this research, relates cultural differences in perception, emotion and motivation to specific forms of self-construal. The self-concept is a socio-cognitive construct used to denote all the knowledge people have about their self [1]. The self represents the most important set of cognitive representations available to a person, acting as an information processor, and mediating perception of the world. It filters, interprets and evaluates all the incoming stimuli in terms of their contribution to the individual's well-being.

Individuals of different cultures have different conceptions of the self, modulated on a continuum which varies according to the relationship between the self and others [18]. The interdependent self is grounded in one's connection with relevant others, whereas the independent self is grounded in autonomy, stability and uniqueness. Other people are still important to the independent self, but mainly for social comparison, remaining external to the self. In Eastern cultures (e.g., China) people tend to have a more interdependent self, whereas in Western cultures (e.g., Britain and the United States) people tend to have a more independent self. This theoretical distinction between independent and interdependent self, is explicit in several other cultural conceptualizations, including Hofstede's [9] cultural value dimension of collectivism versus individualism. This dialectic conception of self has important implications for the way people present themselves to others and how they communicate [24]. In particular, people with an interdependent self tend to describe themselves through roles 
and relationships (e.g., Father of X; Daughter of Y). They use an indirect communication style, and prefer to express self-criticism in order to maintain harmony. By contrast, people with an independent self express themselves through their inner thoughts and feelings [13]. These people use a direct communication style as they are driven by the realization of personal goals and the manifestation of individual capabilities.

Another way of framing the influence of the speaker's cultural background on communication style is by distinguishing between low-context and high-context communication cultures [8]. At the basis of this framework is the observation that the meaning of verbal communication often interacts with the context in which it occurs. In some societies, the cultural context in which most interpersonal interaction takes place is very strong due to their homogeneous make-up and long standing cultural traditions. In these societies, which include most Eastern societies, people can rely much more on a shared cultural context, and need not use explicit communication to make themselves understood. Other societies, in contrast, have a much more heterogeneous make-up and shorter cultural traditions. People in these societies, which include most Western societies, need to make their communication very explicit in order to be understood by others.

Differences in the perception of oneself may also have consequences for the way one perceives others. It is suggested that individuals with an interdependent self are more advertent to the needs of other people in order to maintain harmonious relationships, whereas individuals with an independent self care less about others' details due to their focus on themselves [14]. This suggests that the cultural background of the perceiver will determine what information they take into account when judging other people's self-presentations. A study amongst Korean people found that individuals with a more independent self preferred positive presentation styles to negative ones, whereas preferences of individuals with a more interdependent self were the other way around [10].

In summary, the literature on cultural differences suggests that culture affects the way people present themselves to others, the communication styles they use, and how they perceive other people. The study presented in this paper aims to investigate whether cultural differences in people's online self-presentation affect the way in which they are perceived and how this relates to the cultural background of the perceiver. Based on the background literature we developed two hypotheses about online communication:

H1: Differences in communication style and self-presentation in verbal communication will affect people's social perceptions.

H2: People from different cultures will base their opinions of others on different aspects of their self-presentation and communication style.

In order to test these hypotheses, people's social perception of pieces of online communication typical of British people and Chinese people were measured. This was achieved by independently manipulating the blog style and physical appearance embedded in a personal virtual space modeled on Windows Live Spaces. These manipulations created four different virtual spaces: Two in which the appearance matched the cultural style of the blog (congruent), and two in which the appearance did not match the cultural style of the blog (incongruent). These combinations lead to a third hypothesis: 
H3: Congruent Combinations of appearance and blog style will be perceived as more socially desirable than incongruent combinations.

\section{Related Work}

Most of the work related to cultural differences in online communication has concentrated on cultural differences in presentation on Web sites and personal virtual spaces. An inventory of self-presentation on MSN Spaces (the forerunner of Windows Live Spaces) owned by either British or Chinese students studying at British Universities revealed strong cultural differences in line with the distinction between interdependent and independent self [3]. For example, spaces owned by Chinese students conformed more closely to a design standard, featured more extensive friend lists, and contained more pictures. Chinese students were also more accommodating, and more inclined to host filter blogs (blogs composed of filtered feeds from other people's blogs).

Other work has focused on the cues receivers of online communication use to form impressions of the people who produced the communication. As mentioned in the introduction, there may be several strategies for dealing with a lack of cues for social perception in online communication, one of which is a tendency of "Internet users develop impressions of others, even with the limited cues available online, by adapting to the remaining cues in order to make decisions about others. Online users look to small cues in order to develop impression of others, such as a poster's email address, the links on a person' homepage, even the timing of email messages." [4]. These small cues may lead to the activation of stereotypes in an attempt to fill the gaps and create impressions of others that cover many more aspects of their personality then there is evidence for [6]. Of the cues that are available in online communication, a person's appearance is one that could easily trigger this application of stereotypes [16].

\section{Method}

\subsection{Participants}

A total of 80 students participated in the study as volunteers. Half of them were born in the UK and half of them were originally from China but studying in UK. The data obtained from two of the British-born participants were later excluded from the analyses because they indicated that their ethnicity was Chinese. The average age of the British participants was $24.18(\mathrm{sd}=5.60)$, and that of the Chinese participants was 23.93 (sd=3.75). Facebook (http://www.facebook.com) was used as the main social networking platform by $100 \%$ of the British participants, but only by $25 \%$ of the Chinese participants. The other $75 \%$ of the Chinese participants reported using mainly Windows Live Spaces (http://home.spaces.live.com). The gender composition of each group was exactly 50/50. 


\subsection{Apparatus}

The manipulation of communication style was based on the seven dimensions of cultural differences between Western and Eastern societies described in Table 1, which were used to create two blogs. Each blog contained three diary entries reporting different aspects of student life. A British student and a Chinese student wrote the diaries together in English. They were given three hypothetical events, and discussed how they would deal with those events, focusing on their attitudes and behaviours. The first theme was about a supervisor who criticized a student's work. The British student complained about the supervisor by saying "I was miffed because she did not agree with the research topic", whereas the Chinese student showed respect for the supervisor by saying "I appreciate his encouragement and supervision, I need to make every effort to please my supervisor" (e.g., large vs. small power distance [9]). The theme of the second diary entry was the student's birthday. The British student's focus was on herself, "I usually detest birthdays, because they mean I'm getting on ... But who's to care? ... So I went out for lunch to celebrate and had a makeover ...", whereas the Chinese student's focus was on her friends and diverting attention away from herself, "All of a sudden Bill, Catherine, Alex turned up at my house shouting "come and get your present"... I was dumbstruck and forgot to invite them into my house. My house is too small and not very clean" (e.g., individualism vs. collectivism [9]). The theme of the third diary entry was about students working together on a group-work assignment. An attempt was made to make all of the cultural dimensions explicit in the differences between the diaries. Inevitably, this may have introduced other differences, such as language, but this was kept to a minimum by rigorous checks for grammatical correctness. Idiosyncratic expressions were avoided as much as possible without making the diaries sound stifled.

Table 1. Seven Dimensions of Cultural Differences between Eastern and Western Societies

\begin{tabular}{lll}
\hline Eastern Culture & Western Culture & Reference \\
\hline Interdependent & Independent & {$[9],[18]$} \\
Indirect & Direct & {$[8],[18]$} \\
Reserved & Open & {$[8],[18]$} \\
Implicit & Explicit & {$[8]$} \\
Relationship-oriented & Task-oriented & {$[8],[9],[18]$} \\
Hierarchical & Social Equal & {$[9]$} \\
Long-term orientation & Short-term orientation & {$[9]$} \\
\hline
\end{tabular}

The manipulation of the appearance of the presenter was realized by the creation of two profiles: One containing a photo of a typical Caucasian face and typically British name (Emily Sutton), the other containing a photo of a typical Asian face and a typical Chinese name (Song Yang). The photos were selected from Rhodes et al. [22] on the basis that they were typical for their particular ethnic group and judged to be equally attractive. The profiles and blogs were styled on the format used by Windows Live Spaces. Combining the blogs and profiles created four personae: two with a congruent combination of blogs and profiles (e.g., Chinese style blog with Chinese 
appearance), and two with an incongruent combination of blogs and profiles (e.g., British style blog with Chinese appearance) ${ }^{1}$.

\subsection{Instruments}

The perception of personae was measured in terms of the seven cultural dimensions of Table 1, the Interpersonal Attraction Scale [20] and the Source Credibility Scale [19]. Ratings on the cultural dimensions were obtained through five-point semantic differential scales between two bipolar adjectives with opposite meanings at each side (e.g., between 'direct' and 'indirect'). The Source Credibility Scale was designed to measure the extent to which a person was deemed to possess Competence (e.g., "unintelligent-intelligent"), 'Caring/Goodwill' (e.g., "self-centred-not self-centred"), and 'Trustworthiness' (e.g., "unethical-ethical”). Each subscale had six items which were also rated on five-point semantic differential scales. The Interpersonal Attraction scale was designed to measure a person's perceived Physical Attractiveness (e.g., "I think she is quite pretty"), Social Attractiveness (e.g., "I would like to have a friendly chat with her"), and Task Attractiveness (e.g., "you could count on her to get the job done"). Each subscale had six items which were rated on a 5-point Likert-type rating scale (1=strongly disagree, $5=$ strongly agree).

\subsection{Procedure}

Each participant was given one of the combinations of blogs and profile, and given 20 minutes to read the content of the blog and look at the profile. Immediately afterwards they were asked to fill out a questionnaire which included questions about their age, country of origin and online social networking habits, rating scales for the seven cultural dimensions, the Interpersonal Attraction Scale and Source Credibility Scale. A semi-structured interview was then conducted to further assess participants' perception of the persona (e.g. "where do you think the persona is originally from?") and their general experiences in on-line social networking. The total time spent by each participant was approximately 40 minutes.

\section{Results}

Multivariate Analysis of Variance (MANOVA) was used to test the effects of the independent variables on perceptions of the personae along the cultural dimensions. Univariate Analysis of Variance (ANOVA) was used to evaluate the effect of the independent variables on the scores on the subscales of the Interpersonal Attraction Scale and Source Credibility Scale. Scores on all of the subscales were calculated by adding the ratings on the six items making up each of the scales, as recommended by the original authors. As a result, the scores ranged from 6 (ratings of 1 on all six items) to 30 (ratings of 5 on all six items). Partial eta squared statistics (partial $\eta^{2}$ )

\footnotetext{
${ }^{1}$ The examples of a congruent persona with a Chinese style blog and a Chinese appearance and a incongruent persona with a British style blog and a Chinese appearance are available in the following URLs: http://hiyahiyahiya1983.spaces.live.com/, http://goodbyemylover4ever.spaces. live.com/
} 
were used as estimates of effect size. Partial $\eta^{2}$ was computed considering the variance attributable to the effect of interest plus error [21]. As a general guideline, $\eta^{2}=$ .01 is considered small, $\eta^{2}=.06$ medium, and $\eta^{2}=.14$ large. Post-hoc tests for investigating significant interaction effects were done using Tukey HSD which controls the experiment-wise type I error [12]. Pearson's correlation coefficient was used to calculate the relationships between sub-scales and those between cultural dimensions and sub-scales.

\subsection{Experimental Manipulation Check}

The internal consistency of the seven cultural dimensions was measured as alpha $=0.76$ and all corrected-item correlation were larger than 0.38. It appears, therefore, that there is an underlying construct being measured by these dimensions. Multivariate analysis of participants' ratings on the cultural dimensions revealed a significant effect of Blog Style $\left(\mathrm{F}(7,64)=24.00, p<.001\right.$, partial $\left.\eta^{2}=.72\right)$ (Fig. 1). No other effects were significant. British blogs scored higher on all seven cultural dimensions (all $p s<.001$ ). Hence we can conclude that participants were able to distinguish between the blogs based on stereotypical cultural characteristics of their communication style. The other experimental manipulation involved the physical appearance, in which the independent variables on ratings of physical attractiveness of the personae were tested. No significant effects were found which ensured that physical attraction did not have to be considered in the interpretation of the effects on other variables found in this experiment.

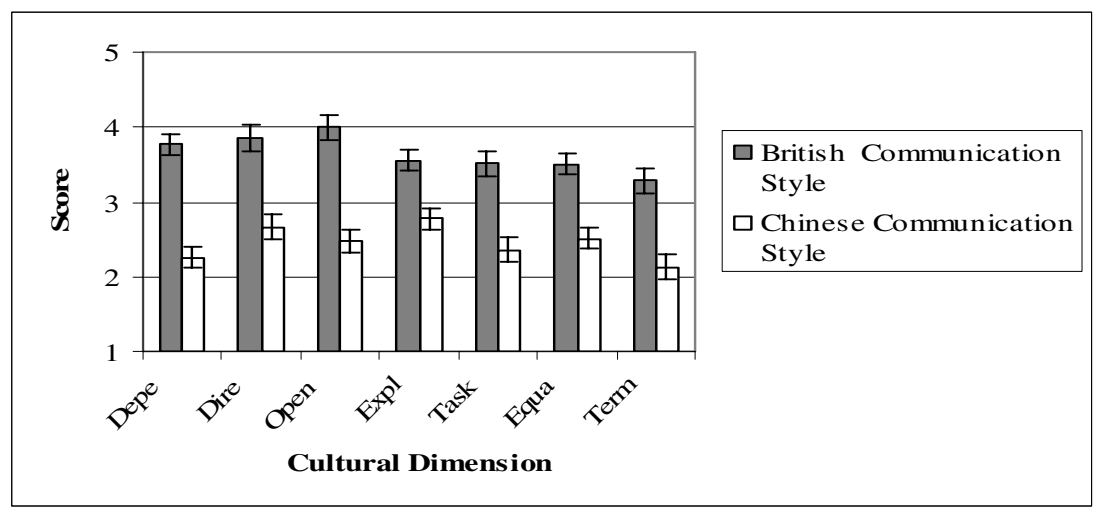

Fig. 1. Means ratings of the cultural dimensions for personae with British and Chinese communication styles (Error bars show the standard error)

Overall it was important to see if there was agreement amongst participants when attributing an origin to the persona they were asked to judge, and particularly how appearance and blog style affected this attribution. Table 2 shows the results. It can be seen that almost all participants thought that the congruent personae with British and Chinese appearances had their origins in the UK/Europe and China/Asia respectively. 
However, their opinions about the origins of the incongruent personae were more diverse. In particular, the persona with British blog style and Chinese appearance was regarded by most Chinese participants and some British participants as a British Born Chinese (BBC) or a person from Hong Kong. In this case, appearance was more important than blog style in judging a person's origin for most British participants, while most Chinese participants took both appearance and blog style into account. This may be due to a lack of knowledge in the differences that exist between Chinese people, from different parts of China, from the British participants.

Table 2. Judgments about the Origins of the Personae (* BBC and people from Hong Kong were clustered together because they have a Chinese appearance but reflect behaviour that is more typical of British culture)

\begin{tabular}{|c|c|c|c|c|c|}
\hline \multirow[b]{2}{*}{$\begin{array}{l}\text { Blog/Appearance } \\
\text { Combination }\end{array}$} & \multirow[b]{2}{*}{$\begin{array}{l}\text { Participant's } \\
\text { Origin }\end{array}$} & \multicolumn{4}{|c|}{ Attributed Origin } \\
\hline & & UK/Europe & China/Asia & $\begin{array}{l}\text { BBC/Hong } \\
\text { Kong }\end{array}$ & Other \\
\hline Congruent & British & 10 & & & \\
\hline $\begin{array}{l}\text { (British blog style; } \\
\text { British appearance) }\end{array}$ & Chinese & 10 & & & \\
\hline Incongruent & British & & 5 & 4 & \\
\hline $\begin{array}{l}\text { (British blog style; } \\
\text { Chinese appearance) }\end{array}$ & Chinese & 1 & 2 & 7 & \\
\hline Congruent & British & & 9 & & 1 \\
\hline $\begin{array}{l}\text { (Chinese blog style; } \\
\text { Chinese appearance) }\end{array}$ & Chinese & & 10 & & \\
\hline Incongruent & British & 7 & & & 2 \\
\hline $\begin{array}{l}\text { (Chinese blog style; } \\
\text { British appearance) }\end{array}$ & Chinese & 8 & 1 & & 1 \\
\hline
\end{tabular}

\subsection{Test of Hypotheses}

Overall, participants' answers exhibited high reliability. The Cronbach alpha reliability for the Interpersonal Attraction Scales was calculated as 0.85 for Social Attraction, 0.80 for Physical Attraction and 0.80 for Task Attraction. The Cronbach alpha reliability for the Source Credibility scales was calculated as 0.71 for Competence, 0.73 for Caring/Goodwill, and 0.76 for Trustworthiness.

\subsubsection{Social Attraction}

There was a significant main effect of Blog Style on participants' judgment of Social Attraction $\left(\mathrm{F}(1,70)=4.27, p<.05\right.$, partial $\left.\eta^{2}=.05\right)$. There was also a significant two-way interaction between Nationality and Blog Style $(\mathrm{F}(1,70)=11.35, p<.01$, partial $\eta^{2}=.14$ )(Fig. 2). Post-hoc analysis revealed that the British blog style was judged more socially attractive by British than by Chinese participants. In addition, Chinese participants who judged personae with a British blog style gave lower ratings than those who judged personae with a Chinese style $(p s<.01)$. No such difference was found between groups of British participants. The judgment of Chinese participants appeared to be affected by blog style, but not the judgment of British participants. 


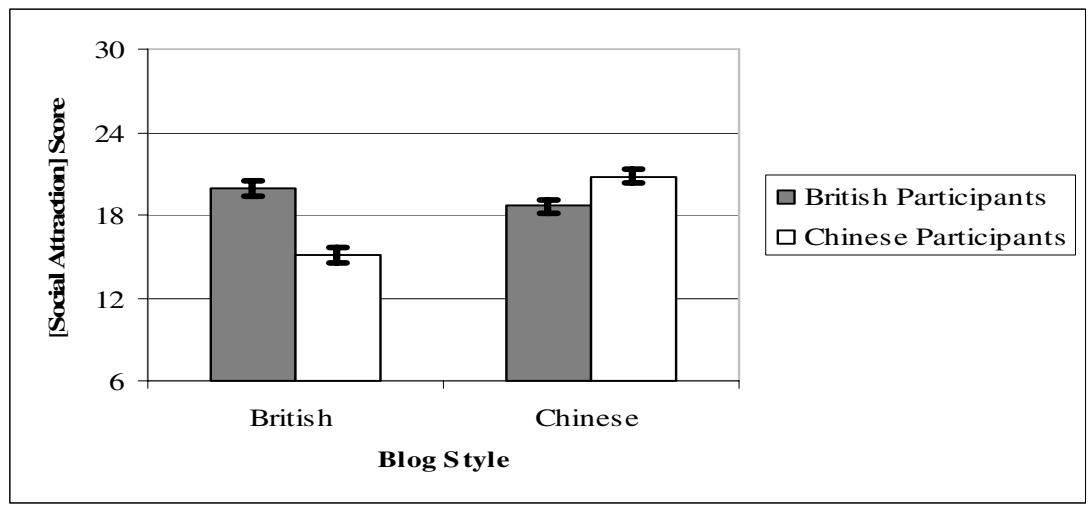

Fig. 2. The two-way interaction between Nationality and Blog Style on the perception of "Social Attraction" (Error bars show the standard error)

\subsubsection{Task Attraction}

There was a significant effect of Blog Style on participants' judgments of "Task Attraction" $\left(\mathrm{F}(1,70)=5.26, p<.05\right.$, partial $\left.\eta^{2}=.07\right)$. There was also a significant two-way interaction effect between Blog Style and Appearance $(\mathrm{F}(1,70)=4.53, p<.05$, partial $\left.\eta^{2}=.06\right)($ Fig. 3). Post-hoc analysis revealed that the incongruent persona with a Chinese appearance and British blog style received higher scores on this scale than the congruent persona with a Chinese appearance and Chinese blog style $(p<.05)$. No difference was found in the scores received by the personae with a British appearance.

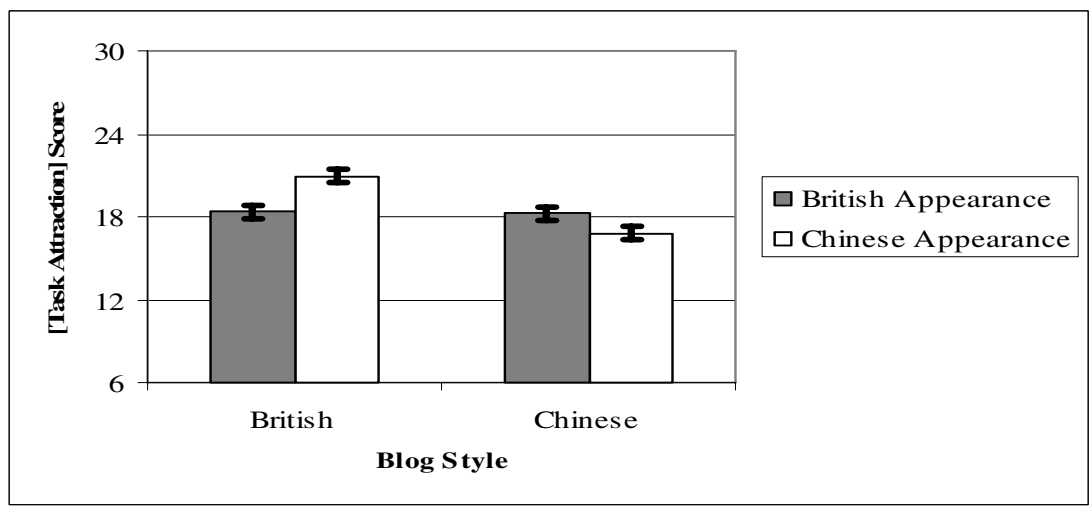

Fig. 3. The two-way interaction effect of Blog Style and Appearance on the perception of the "Task Attraction" (Error bars show the standard error)

\subsubsection{Competence}

There was a significant effect of Blog Style on participants' judgments of "Competence" $\left(\mathrm{F}(1,70)=32.03, p<.01\right.$, partial $\left.\eta^{2}=.31\right)$. There was also a significant three-way interaction among Nationality, Blog Style and Appearance $(\mathrm{F}(1,70)=4.01, p<.05$, partial $\left.\eta^{2}=.05\right)$ (Fig. 4). Post-hoc analysis revealed that the congruent persona with a 
British blog style and British appearance received higher scores than the incongruent persona with a British blog style but Chinese appearance when rated by British participants $(p<.01)$. The congruent persona with Chinese blog style and Chinese appearance received lower scores than the incongruent persona with British blog style and Chinese appearance when rated by Chinese participants $(p<.05)$. This suggests that British participants based their perception of competence on both appearance and communication style of online persona, whereas verbal cues had a bigger impact on the ratings of Chinese participants.

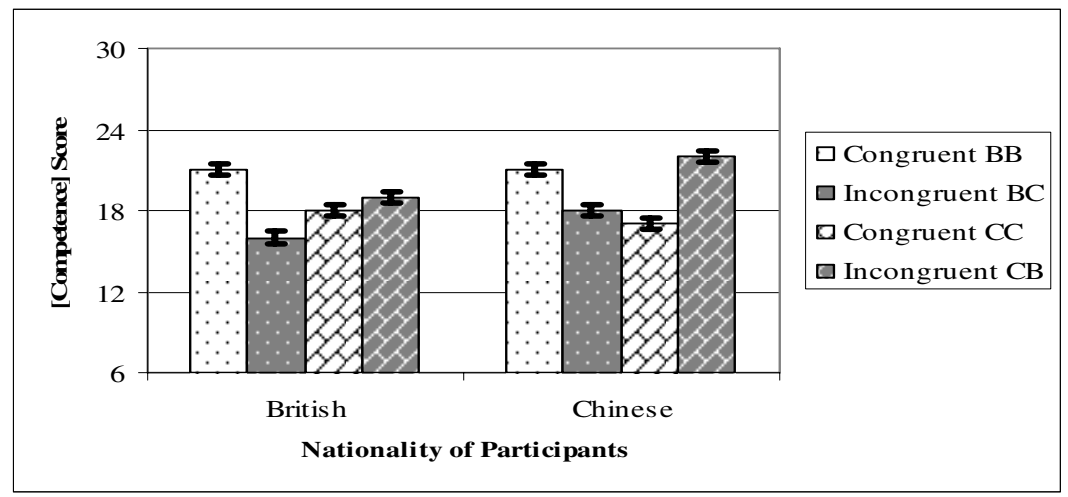

Fig. 4. The three-way interaction effect on the perception of the "Competence" of the personae (Error bars show the standard error)

\subsubsection{Caring/Goodwill}

There was a significant effect of Blog Style on participants' judgment of "Caring/Goodwill" $\left(\mathrm{F}(1,70)=23.89, p<.01\right.$, partial $\left.\eta^{2}=.25\right)$. There was a significant twoway interaction between Nationality and Blog Style $(\mathrm{F}(1,70)=5.10, p<.05$, partial $\eta^{2}=.06$ )(Fig. 5). Post-hoc Analysis revealed that the goodwill of personae with a

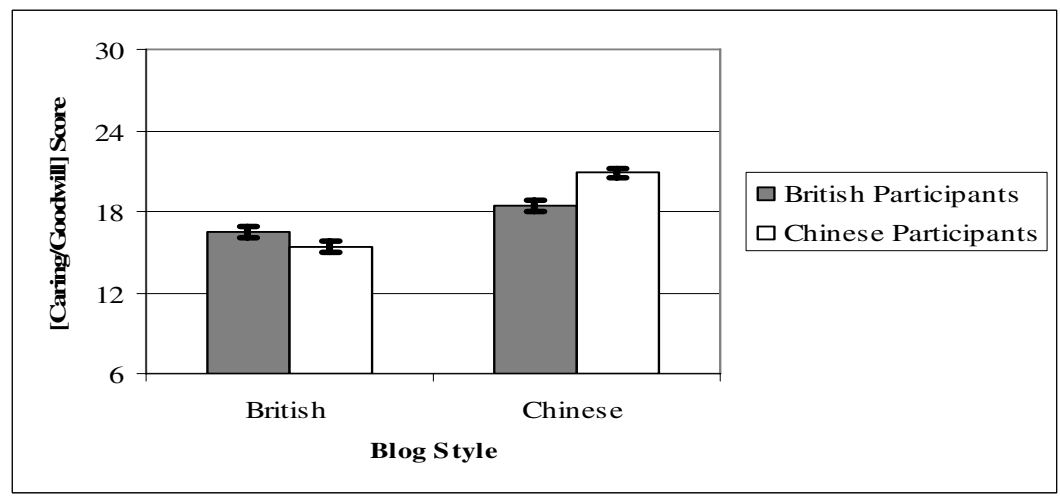

Fig. 5. The two-way interaction effect on the perception of the "Caring/Goodwill" of the personae (Error bars show the standard error) 
Chinese presentation style was judged to be higher than that of personae with a British presentation style by Chinese participants $(p s<.01)$, but no such difference was found for British participants. Again the judgments of Chinese participants appeared to be affected by the blog style, but not those of British participants.

\subsubsection{Trustworthiness}

There was a significant three-way interaction among Nationality, Blog Style and Appearance $\left(\mathrm{F}(1,70)=5.58, p<.05\right.$, partial $\left.\eta^{2}=.07\right)(\mathrm{Fig}$. 6). Although post-hoc analysis revealed no significant pair-wise differences, there was a trend that congruent personae were rated as more trustworthy than incongruent personae when judged by British participants, whereas a slight opposite trend was observed for Chinese participants.

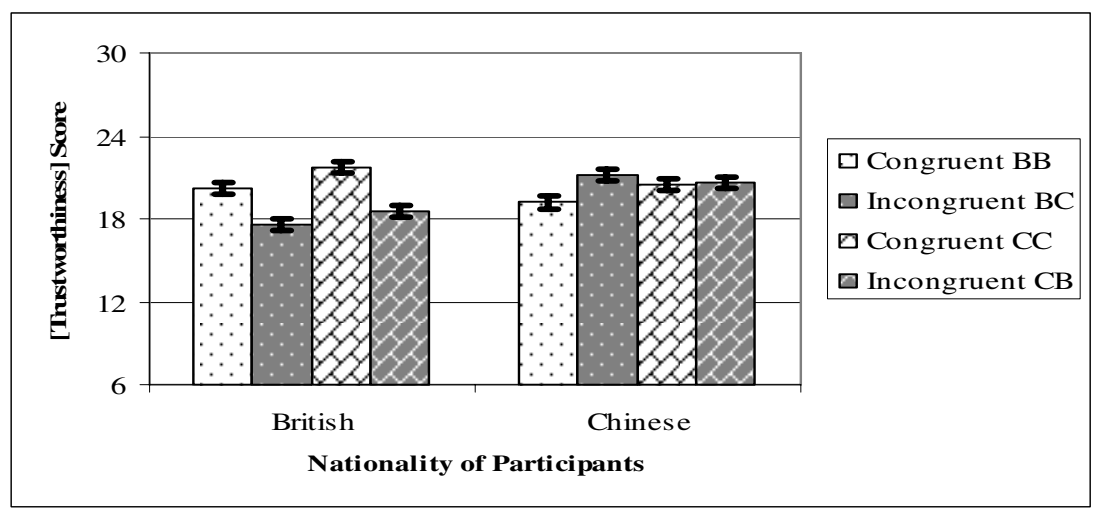

Fig. 6. The three-way interaction effect on the perception of the "Trustworthiness" of the personae (Error bars show the standard error)

\subsection{Correlations}

Correlations were calculated between ratings on the Interpersonal Attraction scales and Source Credibility scales for both British and Chinese participants separately. For British participants there were no significant relationships ( $p s>05$ ) Chinese participants tended to see more competent personae as less socially attractive $(\mathrm{r}=-.49$, $p<.01)$, and preferred making friendships with more caring personae $(\mathrm{r}=.54, p<.01)$. Remarkably, however, physical attraction was somewhat related to task attraction for Chinese participants $(\mathrm{r}=.34, p<.05)$.

Table 3 shows the correlations between ratings of the personae on the seven cultural dimensions and ratings on the Interpersonal Attraction and Source Credibility Scales. From this table it is clear that Chinese participants perceived personae with the British style of communication to be more competent, but also less caring based on all cultural dimensions. Chinese participants also base their judgments of social attractiveness on the interdependence, hierarchy, and relationship and long-term orientation of the blog style. All of these appear more in Chinese style blogs. British participants tended to associate an independent blog style with more competence, an 
explicit communication style with more socially attractive, as well as associated blog styles that were more direct, and more focused on social equality and short-term relations with higher task attractiveness. All of these appear more in British style blogs.

Table 3. Significant Correlations between 7-Cultural Dimensions and Scales among British and Chinese participants $(* *=$ significant at the 0.01 level; * $=$ significant at the 0.05 level)

\begin{tabular}{llll}
\hline Cultural Dimension & Nationality & Scale & $\mathbf{r}$ \\
\hline \multirow{3}{*}{ Interdependent vs. Independent } & British & Competence & $.48^{* *}$ \\
& Chinese & Social Attraction & $-.68^{* *}$ \\
& & Competence & $.52^{* *}$ \\
Indirect vs. Direct & British & Caring/Goodwill & $-.68^{* *}$ \\
& Chinese & Task Attraction & $.34^{*}$ \\
& & Competence & $.35^{*}$ \\
Reserved vs. Open & British & Caring/Goodwill & $-.52^{* *}$ \\
& Chinese & Competence & $.49^{* *}$ \\
& & Caring/Goodwill & $-.48^{* *}$ \\
Implicit vs. Explicit & British & Social Attraction & $.34^{*}$ \\
& Chinese & Competence & $.40^{* *}$ \\
\multirow{2}{*}{$\begin{array}{l}\text { Relationship-oriented vs. Task- } \\
\text { oriented }\end{array}$} & British & Caring/Goodwill & $-.44^{* *}$ \\
& Chinese & Social Attraction & $-.43^{* *}$ \\
& & Competence & $.56^{* *}$ \\
Hierarchical vs. Social Equal & British & Caring/Goodwill & $-.50^{* *}$ \\
& Chinese & Task Attraction & $.33^{*}$ \\
& & Social Attraction & $-.36^{*}$ \\
& & Competence & $.56^{* *}$ \\
Long-term vs. Short-term Rela- & Chinese & Caring/Goodwill & $-.36^{*}$ \\
tions & & Task Attraction & $.50^{* *}$ \\
& & Social Attraction & $-.39^{*}$ \\
& & Task Attraction & $.38^{*}$ \\
& & Competence & $.44^{* *}$ \\
& & Caring/Goodwill & $-.39^{*}$ \\
\hline
\end{tabular}

\section{Discussion}

The nature of the interaction between variation in blog style of presenters and cultural background of audiences was clearly illuminated by this study. In particular, most of the cultural characteristics of the Chinese blog style were deemed by Chinese participants to contribute to creating more socially attractive and caring personae. This is consistent with the first hypothesis, stating that differences in communication style and self-presentation in verbal communication will affect people's social perceptions. Moreover, Chinese participants expressed a preference for interacting with people from their own-social group. It also indicated that the desirability of initiating online relationship can be varied between people from different cultures and that it is based on different aspects of self-presentation and communication style (H2).

There was also a clear interaction between the blog style of personae and their appearance on social perception of the participants, and on perceptions of task attractiveness in particular. Surprisingly, incongruent personae tended to be judged as more attractive to work with than congruent personae. This could be explained by taking into consideration the origins attributed to these personae. In particular, incongruent 
persona with Chinese appearance and British blog style were deemed to be Britishborn Chinese or from Hong Kong. Participants may have applied cultural stereotypes of these people that suggest they are high achievers which would make them more attractive to work with. For example, participants may have used their knowledge of the fact that British born children with Chinese ethnicity tend to achieve very high standards in education (e.g., they have the highest percentage of pupils achieving 5 good GCSEs, which are exams taken at age 16) compared to children from other ethnic groups including White British [25].

\section{Conclusions}

The results of this study provided support for the first two hypotheses, but no unequivocal support for the third hypothesis was found. This suggests that cultural differences embedded in online communication can dramatically impact the impressions it creates in people, and that these impressions depend on their cultural background. Indeed, people from different cultural backgrounds rely on different cues when forming impressions of others. British people may pay less attention to verbal cues than Chinese people, who tend to focus more on the detailed content of communication. The emphasis on individualism apparent in Western cultures may make them more suspicious of inconsistencies between appearance and content, whereas people from Eastern cultures may be prone to explain such anomalies away by evaluating individuals in relation to in-group and out-groups. This result is consistent with Hall's [8] high and low context theory. Effective communication in high context cultures requires a high degree of common ground between presenters and receivers. Furthermore, crosscultural experience may play an important role in the findings. Historically the UK has a vast multi-cultural experience, which may affect British participants' responses. Similarly, the Chinese participants may be influenced by British culture, as they have relocated to the UK. Alternative explanations cannot be ruled-out, however, such as the increasing importance of political correctness in cross-cultural encounters typical of British society.

The results may have a number of implications for the design of social networking platforms. Social networking platforms may need to be designed to satisfy cultural differences, due to what we have shown to be important variances in preferences and presentation amongst users. In addition, people should be made aware of the different ways in which their self-presentation can be interpreted differently by people with different cultural backgrounds. Such awareness may increase the effectiveness of cross-cultural online relationship building. While this study only focuses on the role of cultural differences on influencing the initialization of cross-cultural online relationship, it would also be valuable to analyze their effects in maintaining and developing offline to online relationships.

\section{References}

1. Banaji, M.: The Self in Social Contexts. Annual Review of Psychology 45(1), 297-332 (1994)

2. Chen, Y.W.: Intercultural Friendship from the Perspective of East Asian International Students. China Media Research 2(3), 43-58 (2006) 
3. De Angeli, A.: Cultural variations in virtual spaces design. AI \& Society (to appear)

4. Ellison, N., Heino, R., Gibbs, J.: Managing Impressions Online: Self-Presentation Processes in the Online Dating Environment. Journal of Computer-Mediated Communication 11(2), 415-441 (2006)

5. Ellison, N., Steinfield, C., Lampe, C.: The benefits of Facebook "friends": Exploring the relationship between college students' use of online social networks and social capital. Journal of Computer-Mediated Communication 12(3), 1143-1168 (2007)

6. Fiske, S.T.: Stereotyping, prejudice, and discrimination at the seam between the centuries: Evolution, culture, mind and brain. European Journal of Social Psychology 30, 299-322 (2000)

7. Goffman, E.: The Presentation of Self in Everyday Life. Doubleday, New York (1959)

8. Hall, E.T.: Beyond culture. Doubleday, New York (1976)

9. Hofstede, G.: The Business of International Business is Culture. International Business Review 3(1), 1-14 (1994)

10. Kim, J., Kim, M.-S., Kam, K.Y., Shin, H.-C.: Influence of self-construals on the perception of different self-presentation styles in Korea. Asian Journal of Social Psychology 6, 89-101 (2003)

11. Kimmerle, J., Cress, U.: Group awareness and self-presentation in computer-supported information exchange. International Journal of Computer-Supported Collaborative Learning 3(1), 85-97 (2008)

12. Kirk, R.E.: Experimental Design: Procedures for the Behavioral Sciences. Pacific Grove: Brooks/Cole (1995)

13. Kitayama, S., Markus, H.R., Matsumoto, H., Norasakkunkit, V.: Individual and collective processes in the construction of the self: Self-enhancement in the United States and selfcriticism in Japan. Journal of Personality and Social Psychology 72, 1245-1267 (1997)

14. Kitayama, S., Markus, H.R., Kurokawa, M.: Culture, emotion, and well-being: Good feelings in Japan and the United States. Cognition \& Emotion 14, 93-124 (2000)

15. Lampe, C., Ellison, N., Steinfeld, C.: A familiar Face(book): Profile elements as signals in an online social network. In: Proceedings of Conference on Human Factors in Computing Systems, pp. 435-444. ACM Press, New York (2007)

16. Leary, M.R.: Self Presentation - Impression Management and Interpersonal Behaviour. Boulder, CO. Westview (1996)

17. Lee, H.: Privacy, Publicity, and Accountability of Self-Presentation in an On-Line Discussion Group. Sociological Inquiry 76(1), 1-22 (2006)

18. Markus, H.R., Kitayama, S.: Culture and the self: Implications for cognition, emotion, and motivation. Psychological Review 98, 224-253 (1991)

19. McCroskey, J.C., Holdridge, W., Toomb, J.K.: An instrument for measuring the source credibility of basic speech communication instructors. The Speech Teacher 23, 26-35 (1974)

20. McCroskey, J.C., McCain, T.A.: The measurement of interpersonal attraction. Speech Monographs 41, 261-266 (1974)

21. Pallant, J.: SPSS survival manual. Allen \& Unwin (2007)

22. Rhodes, G., Lee, K., Palermo, R., Weiss, M., Yoshikawa, S., Clissa, P., et al.: Attractiveness of own-race, other-race, and mixed-race faces. Perception 34, 319-340 (2005)

23. Schau, H.J., Gilly, M.C.: We are what we post? Self-presentation in personal web space. Journal of Consumer Research 30(3), 385-404 (2003)

24. Triandis, H.: The self and social behaviour in differing cultural contexts. Psychological review 96(3), 506-520 (1989)

25. UK Department for Education and Skills. National Curriculum Assessments and GCSE/GNVQ attainment by pupil characteristics, in England, 2002 (final) and 2003 (provisional), SFR 04/2004 (2004), http://www.dfes.gov.uk/rsgateway/DB/ SFR/s000448/NPD_sfr_text_Finished3.pdf 